\title{
ATIVIDADE CIENTÍFICA QUALIFICADA NOS PROGRAMAS DE PÓS- GRADUAÇÃO EM ENFERMAGEM: UM ESTUDO BIBLIOMÉTRICO
}

\author{
QUALIFIED SCIENTIFIC ACTIVITY IN NON-GRADUATION PROGRAMS IN \\ NURSING: A BIBLIOMETRIC STUDY
}

\author{
Helca Franciolli Teixeira Reis, Mateus Carmo Santos, Claudio Bispo de Almeida, Alba \\ Benemérita Alves Vilela, Sérgio Donha Yarid
}

Universidade Estadual do Sudoeste da Bahia - UESB

\begin{abstract}
The objective was to analyze the scientific teaching production in the Graduate Programs in Nursing in Brazil that obtained concepts 6 and 7 in the CAPES 2013-2016 evaluation. It is a bibliometric study of the scientific productions of 155 professors of Brazilian Nursing Graduate Programs, collected through lattes curricula, from June to July 2017, after which the descriptive analyzes were carried out. Most of the evaluated teachers were female, with a Nursing background, have no $\mathrm{H}$ index and do not have a productivity grant. However, they concentrate their scientific production on articles, with publications in periodicals with high Qualis (A1 to B1). It is concluded that the Graduate Programs in Nursing in Brazil, which obtained concepts 6 and 7 in the CAPES 2013-2016 evaluation, are composed of a majority of teachers who present qualified scientific production in accordance with the requirements of the Brazilian Postgraduate Policy, but not yet sufficient to guarantee an acquisition of funds that are sufficient for research production.
\end{abstract}

Key words: Nursing. Scientific Production Indicators. Education, Graduate. Scientific and Technical Activities.
Resumo

Objetivou-se analisar a produção científica docente nos Programas de Pós-Graduação em Enfermagem no Brasil que obtiveram conceitos 6 e 7 na avaliação CAPES 2013-2016. Trata-se de um estudo bibliométrico das produções científicas de 155 docentes de Programas de Pós-Graduação em Enfermagem brasileira, coletadas por meio dos currículos lattes, no período de junho à julho de 2017, posteriormente, realizou-se as análises descritivas. A maior parte dos professores avaliados foram do sexo feminino, com formação em Enfermagem, não apresentam índice $\mathrm{H}$ e não possuem bolsa de produtividade, entretanto, concentram sua produção científica em artigos, com publicações em periódicos com Qualis elevados (A1 à B1). Conclui-se que os Programas de Pós-Graduação em Enfermagem no Brasil, que obtiveram conceitos 6 e 7 na avaliação CAPES 2013-2016, são compostos por maioria de docentes que apresentam produção científica qualificada de acordo as exigências da Política de Pós-Graduação Brasileira, porém ainda não suficientes para garantir uma aquisição de verbas que sejam suficientes para produção de pesquisa.

Palavras chave: Enfermagem. Indicadores de Produção Científica. Educação de Pós-Graduação. Atividades Científicas e Tecnológicas. 


\section{Introdução}

Os cursos de Pós-Graduação têm passado por muitas transformações no Brasil, na última década, principalmente em relação a expansão de novos cursos e ao aumento da produção científica, já que acabam sendo responsáveis por parte significativa da pesquisa no país, de maneira a colaborarem para o desenvolvimento intelectual.

Com o desenvolvimento da Pós-Graduação torna-se necessária a existência de sistemas de avaliação que melhor compreendam as diversas áreas e suas peculiaridades. A avaliação dos cursos de Pós-Graduação é uma prática recente no Brasil, e esse tipo de avaliação tem permitido aferir, comparar a qualidade dos cursos e planejar o desenvolvimento e direcionamento das pesquisas nas Instituições de Ensino Superior $(\text { IES })^{1}$.

Nesta perspectiva, estudar a produtividade científica nas Instituições, especialmente naquelas que possuem os Programas de PósGraduação Strictu Sensu e são responsáveis pela formação de pesquisadores, torna-se questão relevante ${ }^{1}$.

Deste modo, as instituições realizam o processo de auto-avaliação e, posteriormente, seguem avaliadas pela Coordenação de Aperfeiçoamento de Pessoal de Nível Superior (CAPES), fundação que realiza a avaliação dos Programas de Pós-Graduação (PPGs), preocupase não apenas com a qualidade dos cursos de Pós-Graduação, mas também com a produção científica e intelectual, geradas por meio dos pesquisadores destes programas, pois as mudanças que vêm ocorrendo, principalmente no âmbito social, exigem novas ações promovidas pelas pesquisas frutos destes programas.

Com base neste cenário de produção e publicação, a CAPES criou no Brasil o sistema de classificação dos periódicos nacionais e internacionais para os pesquisadores que produzem artigos no decorrer de suas carreiras acadêmicas. A classificação é realizada pelos comitês de consultores de cada área de avaliação, com base em critérios previamente definidos pela área e aprovados pelo Conselho Técnico-Científico da Educação Superior (CTC-ES), que buscam refletir a importância dos diferentes periódicos para uma determinada área. Dessa forma, o Qualis mede a qualidade dos artigos e de outros tipos de produção, por meio da análise da qualidade dos veículos de divulgação, ou seja, periódicos científicos. Esta classificação de periódicos é concretizada pelas áreas de avaliação e passa por processo anual de atualização.

Esses veículos são enquadrados em oito estratos indicativos da qualidade, em ordem decrescente de valor, a saber:A1, A2, B1, B2, B3, $B 4, B 5$ e C.Em síntese, A1 é o mais elevado com Fator de Impacto igual ou superior a 3,800; A2 Fator de Impacto entre 3,799 e 2,500; B1 - Fator de Impacto entre 2,499 e 1,300; B2 - Fator de Impacto entre 1,299 e 0,001; B3 a B5 são indexados em bases MEDLINE, SCIELO, LILACS dentre outras, porém sem Fator de Impacto e Qualis C é considerado irrelevante, com peso zero².

Desta maneira, todos os professores inseridos em Programas de Pós-Graduação Stricto Sensu (Mestrado e Doutorado), que possuem como prioridade a pesquisa $\mathrm{e}$ disseminação do conhecimento, devem produzir artigos periodicamente e publicar em periódicos para que estes sejam validados ${ }^{2}$.

Apesar de serem em maior quantidade os Programas de Pós-Graduação em Educação com nota três são poucos expressivos no que se refere à produção científica, e também possuem menor tempo de existência ${ }^{3}$. Desta maneira, analisar a produção científica de Programas com maior pontuação, podem servir de eixo norteador para que programas com notas menores possam se basear, e visualizar os caminhos de ampliação e qualificação de sua produção científica, já que essa produção corresponde a $40 \%$ do peso que corresponde a ficha de avaliação da CAPES para se obter a nota de classificação de cada programa.

De tal modo, a CAPES avalia tais cursos e difunde as notas respectivas de cada programa quadrienalmente, informando o nível de resultados que estes programas alcançam em termos de ações docentes, resultados discentes, orientações, qualidade das dissertações e teses, inserção social, implicações regionais, locais e nacionais, entre intercâmbios e projetos aplicados para a sociedade.

Nesta compreensão o presente estudo, elegeu como questão de pesquisa: Como se encontra a produção científica docente nos Programas de Pós-graduação em Enfermagem no Brasil que obtiveram conceitos 6 e 7 na avaliação CAPES 2013-2016?

Nesse sentido, o objetivo do presente estudo foi analisar a produção científica docente nos Programas de Pós-graduação em Enfermagem no Brasil que obtiveram conceitos 6 e 7 na avaliação CAPES 2013-2016. 


\section{Metodologia}

Trata-se de um estudo bibliométrico, que se configura em uma técnica estatística e quantitativa com a finalidade de apresentar índices de produção e de disseminação do conhecimento cientifico ${ }^{4}$. Embora, a Bibliometria tenha sua maior aplicação nos campos da Ciência da Informação é possível aplicá-la em várias áreas do conhecimento, inclusive na área da Saúde, a fim de explorar o impacto da produção de um determinado campo de conhecimento (Enfermagem, por exemplo), a produção e produtividade de um conjunto de investigadores, por meio da construção de indicadores bibliométricos 5 .

Desta forma, foi realizado a bibliometria na intenção de analisar a produção científica de professores vinculados aos programas de PósGraduação em Enfermagem Brasileiros. Os critérios de inclusão do presente estudo foram os seguintes: Programas de Pós-Graduação em Enfermagem avaliados no período de 2013 a 2016 pela Coordenação de Aperfeiçoamento de Pessoal de Nível Superior (CAPES) com notas6 e 7, e a produção científica compreendida entre os anos de 2012 e 2017.

Dessa forma, foram incluídos no presente estudos seguintes Programas de Pós-Graduação em Enfermagem do Brasil: Programa de PósGraduação Interunida desde Doutoramento em Enfermagem da Universidade de São Paulo (USP); Programa de Pós-Graduação em Enfermagem em Saúde Pública da Escola de Enfermagem de Ribeirão Preto da USP; Programa de PósGraduação em Enfermagem (PEN) da Universidade Federal de Santa Catarina (UFSC); Programa de Pós-Graduação em Enfermagem Fundamental (PPGEF) da USP e Programa de PósGraduação em Enfermagem na Saúde do Adulto (PROESA) da USP.

A coleta de dados ocorreu nos meses de Junho e Julho de 2017 por meio de consultas aos websites dos programas para identificação dos professores. Posteriormente, a coleta de dados foi feita através do acesso ao website (www.lattes.cnpq.br) com um instrumento elaborado com os seguintes aspectos: sexo, formação, bolsa de produtividade, índice $\mathrm{H}$, valor do índice $\mathrm{H}$, quantidade de artigos publicados (2012-2017), livros e capítulos de livros e um item que considerou a qualidade dos artigos publicados de acordo com a estratificação do qualis das revistas nas quais foram publicados.

Os dados foram tabulados em uma planilha do Exce/2010 e posteriormente analisados pelo software Statistical Packageof Social Science (SPSS, versão 20.0). Foram realizadas análises descritivas (média, desvio padrão, frequência absoluta, frequência relativa, mínimo e máximo), com base em autores que também discutiram a temática do presente estudo. O presente estudo não foi submetido ao comitê de ética em pesquisa, já que os dados coletados são de domínio público.

\section{Resultados e Discussões}

Foram coletados dados de 155 professores vinculados aos programas de enfermagem com notas 6 e 7, sendo que 146 (94,2\%) são do sexo feminino e 148 (95,5\%) com formação em Enfermagem e os demais em outras profissões (Odontologia, Educação Física, Medicina, Medicina Veterinária, Fisioterapia). O tempo médio de atuação profissional foi de $30,18( \pm 9,81)$ anos.

Dentre os professores analisados, aproximadamente quatro em cada dez professores, têm bolsa de produtividade e índice $H$ (figura 1). Em relação à bolsa de produtividade, aproximadamente metade (46,3\%) dos professores têm a bolsa 2 de produtividade e apenas $11,9 \%$ tem a $1 \mathrm{~A}$, que é o maior nível entre as bolsas de produtividade.

Figura 1. Distribuição dos professores de acordo com as bolsas de produtividade e índice $H(n=155)$.
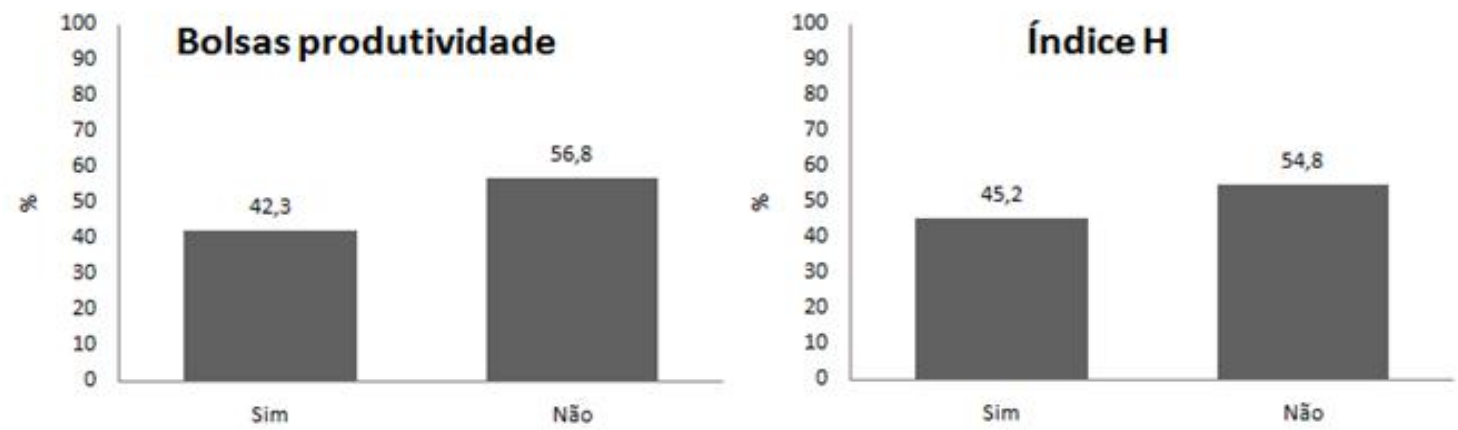
Na Tabela 1 são apresentadas as médias, desvios padrão, mínimo e máximo da produção científica através da publicação de artigos científicos (total e estratificado por Qualis), livros e capítulos de livros. Observa-se que apesar dos artigos estratificados concentrarem sua produção em estratos mais qualificados A2 (8,35\%) e B1 $(8,46 \%)$, nota-se que estratos inferiores (com menor pontuação) B3 a B5 (2,73\%) também são procurados pelos docentes.

Tabela 1. Produção científica dos docentes vinculados aos Programas de Pós-Graduação em Enfermagem(n=155).

\begin{tabular}{ccc}
\hline & Média (dp) & Mínimo-Máximo \\
\hline Livros & $2,66(5,75)$ & $0-60$ \\
Capítulos de Livros & $14,20(17,76)$ & $0-121$ \\
Artigos (total) & $30,81(17,63)$ & $8-131$ \\
Estratificados & & \\
A1 & $4,21(4,30)$ & $0-22$ \\
A2 & $8,35(5,99)$ & $0-25$ \\
B1 & $8,46(7,70)$ & $0-64$ \\
B2 & $5,30(5,04)$ & $0-28$ \\
B3 - B5 & $2,73(2,54)$ & $0-12$ \\
*outros & $1,89(2,26)$ & $0-12$ \\
\hline
\end{tabular}

dp: desvio padrão.

* estratificação C, ou estratificação não identificada.

O sistema brasileiro de avaliação dos Programas de Pós-Graduação Stricto Sensuacaba considerando uma maior pontuação para as produções intelectuais (artigos, livros, capítulos de livro, orientações de teses, dentre outras), tornando o número de publicações como referência quando se pretende avaliar a produção do conhecimento acadêmico. Tal fato conduz os pesquisadores a concentrarem seus esforços acadêmicos para o incremento da produção científica, sobretudo, na publicação de artigos científicos, pois a produtividade em pesquisa é considerada um dos principais itens observados na avaliação para a concessão de financiamentos pelos editais $\mathrm{CNPq}^{6}$.

Na Tabela 1 pode-se perceber a busca pela produtividade no número total de artigos publicados, atingindo uma média de 30,81 artigos por docente no período de cinco anos, concentrados nos periódicos com maior estratificação em seu Qualis (A1, A2 e B1). As publicações em periódicos qualificados têm forte influência na pontuação de Programas de PósGraduação, na busca de verbas para a pesquisa e, dentre outras vantagens acadêmicas, para a aquisição de bolsas, sejam elas de produtividade, de iniciação científica ou mesmo para os discentes dos Cursos de Pós-Graduação Stricto Sensu ${ }^{6}$

Em estudo com Programas de PósGraduação em Educação também foi encontrada uma concentração das produções em artigos (internacionais, nacionais e locais), mesmo com o aumento do número de teses e dissertações ${ }^{2}$.

Vale salientar que a produção científica é apenas uma das dimensões a serem avaliadas, e que o trabalho docente compreende, principalmente, atender à formação dos discentes dos cursos de Pós-Graduação, considerando outros pontos a serem desenvolvidos durante o processo. Entretanto, a necessidade imposta pelo incremento dos incentivos e gratificações institucionais relacionadas à produtividade cientificar e forçam o comportamento produtivista dos docentes ${ }^{6}$.

Outros pesquisadores, na intenção de identificar a raiz do problema que circunda a valorização da construção do conhecimento científico, que eclode no produtivismo para o processo de formação de pesquisadores na PósGraduação, focam seus estudos na percepção do aluno de Graduação em Enfermagem acerca da pesquisa científica, apreendendo, dentre outras considerações, que estes alunos entendem a importância da pesquisa em sua formação científica e profissional, apontando como estímulos para a pesquisa as Instituições de Ensino Superior de qualidade, as atividades de extensão e o apoio docente ${ }^{7}$.

Alguns autores, ao discutirem a formação de pesquisadores em Programas de PósGraduação no Brasil, percebem a interferência do modelo de avaliação da CAPES, juntamente com a política de Pós-Graduação ${ }^{8}$. Tal conformação, acaba não sendo capaz de atender ao esperado dos doutores num cenário nacionale internacional, bem comode intensificação das relações e do intercâmbio científico, econômico e cultural.

Nota-se que o sistema de avaliação CAPES 
acaba homogeneizando os Programas de PósGraduação brasileiros, ao considerar a estratificação pelo Qualis não permite uma autoavaliação pelos Programas ${ }^{2,9}$. Outro ponto a ser considerado é que este modo de avaliação acaba sendo utilizado como um processo de controle, punição ou premiação para os Programas de PósGraduação, ao invés de ser utilizada como um processo integrado a parte pedagógica, científica e administrativa das Instituições de Ensino Superior ${ }^{9}$.

Alguns estudos são unanimes em citar a "produtividade" como algo a ser observado quando se trata do sistema de avaliação da CAPES, visando atender os critérios de qualificação do Estado, bem como propõem que é necessário repensar tais critérios avaliativos $^{2,9,10}$. Em um estudo com enfermeiros pesquisadores, no Brasil e em Portugal, foi indicado que deveriam existir mais indicadores qualitativos na avaliação, pois os autores acreditam ser uma forma de avaliação mais justa e coerente com as necessidades sociais ${ }^{10}$.

Sobre a fragilidade no modelo de avaliação e mensuração quantitativa da produção científica na Saúde Coletiva, pesquisa anterior a esta, ao analisar as subáreas, constatou desigualdades nas medidas da produção científica ${ }^{11}$. Neste caso, destaca a busca incessante do quantitativo de produções científicas, incentivando o produtivismo e ampliando as iniquidades entre os Programas das diferentes subáreas, não considerados mais aspectos qualitativos no modo de avaliação.

Ao discutir sobre as normalizações que mesuram a produtividade acadêmica de pesquisadores e Instituições utilizadas pelas agências de fomento, Café, Ribeiro e Ponczek ${ }^{12}$ consideram que tanto instituições nacionais quanto as estrangeiras, estão submetidas a este processo de avaliação direcionado para a pósgraduação ${ }^{12}$. Desta forma, percebe-se que a busca pela produtividade é um fenômeno que envolve a ciência mundial na atualidade.

No presente estudo, o número de bolsistas de produtividade mostra uma preocupação com a qualidade das produções científicas pelos docentes dos Programas de Pós-Graduação estudados. Em relação aos estratos de publicação nos periódicos, resultados similares foram encontrados em um estudo sobre o perfil de bolsistas de produtividade do CNPq na área de Enfermagem, sendo que os artigos produzidos apresentaram maiores prevalências de publicações nos estratos de Qualis $A 2$ e $B 1^{13}$. Já, em nosso estudo, ressalta-se que os dados não foram somente dos bolsistas de produtividade, mas de todos os docentes dos cursos pesquisados.

Outra similaridade nos resultados é em relação ao tipo de bolsa de produtividade, no presente estudo foi encontrado um número maior de bolsistas da categoria 2 em produtividade $(46,3 \%)$, já em outro estudo encontrou-se um predomínio de 63,44\% na mesma categoria ${ }^{13}$. Em relação aos objetivos desta bolsa de produtividade, alguns pesquisadores refletem ser esta, simultaneamente, um prêmio aos cientistas de qualidade e um incentivo à produção de qualidade de tais cientistas ${ }^{14}$. Fato que consideramos causar impactos diferentes nos cientistas e em suas ações, que muitas vezes preocupam-se em alcançar uma maior quantidade em suas produções do que qualidade.

Neste estudo (Figura 1) identificamos que existe um número considerável de docentes como bolsistas de produtividade (42,3\%) e que apresentam o índice $\mathrm{H}(45,2 \%)$. Mesmo sendo menos da metade, tais números indicam uma preocupação dos docentes em produzir de forma competitiva e de acordo com o que prevê a Política Nacional de Pós-Graduação, mais uma vez, priorizando a produtividade acadêmica.

$\mathrm{O}$ índice $\mathrm{H}$ acaba tornando-se uma das principais formas de avaliação do desempenho científico dos pesquisadores em geral, a produtividade em artigos e o seu impacto baseado em citações ${ }^{6}$. Mesmo com limitações, o índice $\mathrm{H}$ é um dos instrumentos utilizados para avaliar a produção científica de pesquisadores ${ }^{15}$.

Em outra área, foi realizado um estudo para identificar o perfil de bolsistas de produtividade em pesquisa CNPq em Psicologia ${ }^{15}$. Os resultados de tal estudo, em relação a bolsistas de produtividade, sinalizaram predomínio do sexo feminino (63\%) e predominância do bolsista tipo 2 (58,6\%). Logo, ambos corroboram com os resultados do presente estudo.

Portanto, compreendemos que, no Brasil, as diversas áreas encontram-se submetidas aos mesmos critérios de avaliação de sua produção científica e dos Programas de Pós-Graduação, levando diversos cientistas a se enquadrarem nas condições de produtividade científica incentivadas pela CAPES, em que o incremento a produção científica é uma realidade evidente, mas que nem sempre está vinculada a qualidade de tal produção.

\section{Conclusões}

Conclui-se que os docentes pesquisadores inseridos em Programas de Pós-Graduação em 
Enfermagem no Brasil, que obtiveram conceitos 6 e 7 na avaliação CAPES 2013-2016 apresentam, em sua maioria, uma produção científica qualificada importante, publicações em periódicos mais bem qualificados ( $\mathrm{A} 1$ à $\mathrm{B} 1$ ), com base nas exigências da Política de Pós-Graduação Brasileira, porém ainda não suficientes para garantir uma aquisição significativa de verbas para a pesquisa, como, por exemplo, grande número de bolsas de produtividade.

Entretanto, tais resultados devem ser vistos com cautela, tendo em vistas que os critérios estabelecidos pelo sistema CAPES avaliam a qualidade da produção científica de acordo com seus estratos e pelo quantitativo da produção, não sendo garantia da aplicabilidade e relevância de muitos estudos.

\section{Referências}

1. Osorio, HP. A produção científica docente nos programas de pós-graduação no Brasil: um estudo bibliométrico. Dissertação (Mestrado em Ciência da Informação) Faculdade de Filosofia e Ciências, Universidade Estadual Paulista, 2012. Disponível em: https://www.marilia.unesp.br/Home/Pos-

Graduacao/Cienciadalnformacao/Dissertacoes/O sorio $\mathrm{H}$ de $\mathrm{P}$ mestrado $\mathrm{Cl}$ 2012.pdf. Acesso em: 02/06/17.

2. CAPES. Classificação da Produção Intelectual. Coordenação de Aperfeiçoamento de Pessoal de Nível Superior. 2016. Disponível em:< http://www.capes.gov.br/avaliacao/instrumento s-de-apoio/classificacao-da-producao-intelectual $>$ Acesso em: jul. 2017.

3. Guimarães IP,Bulhões RS, Hayashi CRM, HayashiMCPI. Avaliação da Pós-Graduação em educação do Brasil: como superar a imprecisão que reina entre nós? Quaestio, Sorocaba, SP, v. 17, n. 1, p. 87-119, maio 2015.

4. Quevedo-Silva F, Santos EBA, Brandão V, Marcelo ML. Estudo Bibliométrico: Orientações Sobre sua Aplicação. Brazilian Journal of Marketing - BJM RevistaBrasileira de Marketing ReMark Vol. 15, N. 2. Abril/Junho. 2016

5. Marcelo JF, HayashiMCPI. Estudo bibliométrico sobre a produção científica no campo da sociologia da ciência. Inf. Inf., Londrina, v. 18, n. 3, p. 138 - 153, set./dez. 2013. http:www.uel.br/revistas/informacao/.

6. Vitor-Costa $M$, Silva $M$, SorianoJB. A avaliação da produtividade em pesquisa na Educação Física: reflexões sobre algumas limitações dos indicadores bibliométricos. Rev. Bras. Educ. Fís. Esporte, São Paulo, v.26, n.4, p.581-97, out/dez, 2012.

7. Oliveira MP, SilvalCM, Albuquerque GC. Pesquisa científica no curso de Enfermagem: revisão integrativa. Revista Práxis, v. 8, n. 16, dez., 2016.

8. Ramos MY, Velho L. Formação de doutores no Brasil: o esgotamento do modelo vigente frente aos desafios colocados pela emergência do sistema global de ciência. Avaliação, Campinas; Sorocaba, SP, v. 18, n. 1, p. 219-246, mar. 2013.

9. Campos VTB, BORGES MF, AraújoJB. Programa de acompanhamento e avaliação da capes: qualidade acadêmica ou controle do estado. Revista Educação e Políticas em Debate - v. 3, n.1 - jan./jul. 2014.

10. Lino MM, Backes VMS, Da Costa MASMC, Martins MMFPS, Lino MM.Influências capitalistas na produção do conhecimento em enfermagem. Rev Gaúcha Enferm. 2017 mar;38(1):e61829. Acesso em: 13 de julho de 2017. Disponível em: http://www.scielo.br/pdf/rgenf/v38n1/01026933-rgenf-1983-144720170161829.pdf

11. IriartJAB, DeslandesSF, Martin D, Camargo Jr KR, Carvalho MS, CoeliCM. A avaliação da produção científica nas subáreas da Saúde Coletiva: limites do atual modelo e contribuições para o debate. Cad. Saúde Pública, Rio de Janeiro, 31(10):2137-2147, out, 2015. Acesso em: 13 de julho de 2017. Disponível em:http://www.scielo.br/pdf/csp/v31n10/0102311X-csp-31-10-2137.pdf

12. Café ALdaP, Ribeiro NM, PonczekR L.A fabricação dos corpos dóceis na pós - graduação brasileira: em cena o produtivism o acadêmico. Encontros Bibli: revista eletrônica de biblioteconomia e ciência da informação 20172275-88. Disponible en: http://www.uacm.kirj.redalyc.redalyc.org/a rticulo.oa?id=14750845007. Fecha de consulta: 12 de Julie de 2017.

13. Santos WM dos, Padoin SMM, Lacerda MR, Gueterres EC. Perfil dos pesquisadores bolsistas de produtividade em pesquisa na área da Enfermagem. Revenferm UFPE online., Recife, 9(supl. 2):844-50, fev., 2015. Acesso em: 13 de julho de 2017. Disponível em: https://www.researchgate.net/profile/Wendel_D os_Santos3/publication/283487723_6018-

68399-1-

PB/links/563a0e6408aeed0531dc9680/601868399-1-PB.pdf?origin=publication_detail.

14. Wainer J, Vieira P. Avaliação de bolsas de produtividade em pesquisa do CNPq e medidas bibliométricas: correlações para todas as grandes áreas. Perspectivas em Ciência da Informação, v.18, n.2, p.60-78, abr./jun. 2013. 
15. Barreto ML, AragãoE, Sousa LEPF de, Santana TM, Barata RB. Diferenças entre as medidas do Índice-H geradas em distintas fontes bibliográficas e engenho de busca. Rev Saúde Pública 2013;47(2):231-8.Acesso em: 13 de julho de 2017. Disponível em: http://www.scielo.br/pdf/rsp/v47n2/0034-8910rsp-47-02-0231.pdf

16. Wendt GW, LisboaCSM, SousaDAA,Koller $\mathrm{SH}$. Perfil dos Bolsistas deProdutividade em Pesquisa do CNPQ em Psicologia. Psicologia Ciência e Profissão, vol. 33, núm. 3, 2013, pp. 536-547.

\section{Endereço para Correspondência}

Universidade Estadual do Sudoeste da Bahia UESB

Rua José Moreira Sobrinho, s/n Campus de -

Jequiezinho, Jequié - BA

CEP.: 45205-490

e-mail:cbalmeida@uneb.br

Recebido em 03/11/2018

Aprovado em 12/01/2019

Publicado em 18/03/2019 